\title{
Guidance on current good radiopharmacy practice for the small-scale preparation of radiopharmaceuticals using automated modules: a European perspective
}

\author{
Joel Aerts, ${ }^{a, b}$ James R. Ballinger, ${ }^{c}$ Martin Behe, ${ }^{d}$ Clemens Decristoforo, ${ }^{e}$ \\ Philip H. Elsinga, ${ }^{f}$ Alain Faivre-Chauvet, ${ }^{g}$ Thomas L. Mindt, ${ }^{\mathrm{h}}$ \\ Petra Kolenc Peitl, ${ }^{i}$ Sergio C. Todde, ${ }^{j}$ and Jacek Koziorowski ${ }^{k *}$
}

This document is meant to complement Part B of the EANM 'Guidelines on current good radiopharmacy practice (cGRPP) in the preparation of radiopharmaceuticals' issued by the Radiopharmacy Committee of the European Association of Nuclear Medicine, covering small-scale in-house preparation of radiopharmaceuticals with automated modules. The aim is to provide more detailed and practice-oriented guidance to those who are involved in the small-scale preparation of radiopharmaceuticals, which are not intended for commercial purposes or distribution.

Keywords: radiopharmaceutical; positron emission tomography; single-photon emission computed tomography; process validation; automated radiosynthesis module

\section{Definitions (see also references 1 and 2)}

Radiopharmaceutical

A radiopharmaceutical is any medicinal product which, when ready for use, contains one or more radionuclides (radioactive isotopes) included for a medicinal purpose.

\section{Small-scale radiopharmaceutical}

A small-scale radiopharmaceutical is any in-house radiopharmaceutical not intended for commercial purposes or distribution, prepared on a small scale, excluding preparations based on licensed generators and labelling kits, and excluding preparation of kits.

Finished product

A finished product is a medicinal product that has undergone all stages of production, including packaging in its final container and labelling.

\section{Preparation}

Preparation includes all operations involved in the purchase of materials and products, production, QC, release and storage of a medicinal product and the related controls.

\section{Small-scale radiopharmacy}

A small-scale radiopharmacy is a facility where small-scale preparation of radiopharmaceuticals is carried out in accordance with national regulations for in-house use. The term small-scale radiopharmacy is not related to the physical size of the facility.

\section{aUniversity of Liege, 4000 Liege, Belgium \\ ${ }^{b}$ University of Paris VII Diderot, 75018 Paris, France \\ 'Guy's and St Thomas' Hospital, London SE1 9RT, UK \\ ${ }^{d}$ ETH-PSI-USZ Paul-Scherrer-Institute, 5232 Villigen-PSI, Switzerland}

eInnsbruck Medical University, 6020 Innsbruck, Austria

fUniversity Medical Center, University of Groningen, 9700 RB Groningen, Netherlands

${ }^{9} \mathrm{CHU}$ de Nantes, 44093 Nantes, France

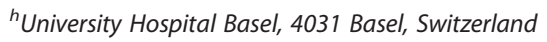

'University Medical Centre Ljubljana, 1000 Ljubljana, Slovenia

jUniversità di Milano-Bicocca, 20052 Monza, Italy

kUniversitetssjukhuset, 58185 Linköping, Sweden

${ }^{*}$ Correspondence to: Dr Jacek Koziorowski, Universitetssjukhuset, Radiofysikavd, 58185 Linköping, Sweden.

E-mail: jacek.koziorowski@lio.se

Abbreviations: CFU, colony forming unit; CGRPP, current good radiopharmacy practice; EANM, European Association of Nuclear Medicine; GMP, good manufacturing practice; HPLC, high-performance liquid chromatography; $Q Q, O Q$, Installation and Operational Qualifications; Ph.Eur., European pharmacopoeia; PV, Process Validation; QC, quality control; SOP, standard operating procedure; SPE, solid-phase extraction; SSRP, small-scale in-house radiopharmaceutical; URS, User Requirement Specification. 


\section{Good radiopharmacy practice}

Good radiopharmacy practice is described in the 'Guidelines on current good radiopharmacy practice (cGRPP)' issued by the Radiopharmacy Committee of the EANM. ${ }^{1}$

\section{Automated module}

An automated module is a device able to perform automatically a sequence of chemical manipulations needed in the preparation of radiopharmaceuticals. An automated module can be commercial or custom made. It consists of two assembled parts: a mechanical part and a chemistry part.

The mechanical part consists of an assembly of electric and/or pneumatic, linear and/or circular actuators, power supplies, pumps, coolers, heaters, sensors for monitoring different parameters (such as temperature, pressure, flow, radioactivity) or any other physical device not in direct contact with chemicals.

The chemistry part is an interconnected network of containers in which gaseous, liquid and/or solid reagents and components can be moved, mixed and/or transformed to obtain the desired final product. The mechanical part and the chemistry part are connected to each other. The contact between the two parts can be permanent (non-disposable device) or temporary (disposable device; 'cassette').

\section{Control system, software and sequence}

Control system controls the automated module (manages and regulates the behaviour) and provides a user interface by which the operator interacts with the module.

Software governs the control system and is generally provided by the module manufacturer and not alterable by users. It is module specific but not necessarily process specific.

Sequence: a list of instructions that the software executes to control the various steps carried out during the preparation of a radiopharmaceutical. A sequence is process specific. Sequences may be changed by the user if the software allows it.

\section{Cassette}

A cassette is a pre-assembled network of containers, valves and syringes, intended to be mounted on the mechanical part of an automated module, becoming then the chemistry part of the module. Cassettes are generally conceived as disposables. A cassette can either be empty or filled with reagents.

\section{Bulk radiopharmaceutical solution (for dispensing)}

The non-sterilized or sterile filtered radiopharmaceutical solution, which is not necessarily isotonic or adjusted for $\mathrm{pH}$ or volume.

\section{Final radiopharmaceutical solution}

The final solution of the radiopharmaceutical compound which is $\mathrm{pH}$ and volume adjusted and sterilized to be ready for injection. It may be intended as a single-dose or multi-dose solution.

\section{Qualification, validation and validation run}

Qualification of equipment is the action of proving that the production process works correctly and actually leads to the expected results. Qualification is part of validation, but the individual qualification steps alone do not constitute process validation.
Validation is the action of proving that any procedure, process, equipment, material, activity or system actually leads to the expected results.

The preparation of radiopharmaceuticals with an automated module must be validated for human use. This includes module $\mathrm{IQ}$, module $\mathrm{OQ}$ and $\mathrm{PV} .^{3}$ In the case of radiopharmaceutical preparation, PV, often called 'validation run', has to be considered as a more general principle than what is typically known as 'performance qualification' and cleaning validation, (e.g. in case of using non-cassette systems, if applicable).

\section{Closed and open operation}

A closed operation is a step in the preparation operation workflow that does not allow any contact between intermediate or final products with external environment. An open operation is a step that allows direct contact between intermediate or final product with external environment (e.g.using an open vial) or allows indirect contact by introduction of an external item in the intermediate or final products (e.g.the introduction of a needle through a septum in a vial). Any open aseptic operation should be evaluated by a risk assessment unless it is performed in a class $A$ environment (e.g. the penetration of a septum by a needle in air class C).

\section{Single vial filling}

A single vial filling is the filling of a vial using a pre-assembled set (filter, needle and vial) without movement of any part of the assembly relative to other parts. Single vial filling is a closed operation. Pre-assembling of material for a single vial filling is an open operation.

\section{Single-use/disposable material}

Single-use or disposable material is any material (including reagents) that is used for the preparation of one unique batch of radiopharmaceutical and is discarded afterwards.

\section{Multiple-use/non-disposable material}

Multiple-use or non-disposable material (e.g. the chemistry part in permanent contact with the mechanical part, multiple-use reagent stock solutions, etc.) is any material that is used for the preparation of successive batches of radiopharmaceuticals.

\section{General aspects and scope}

This EANM guidance covers the preparation of small-scale radiopharmaceuticals with automated modules. It concerns the preparation of radiopharmaceuticals that are not intended for commercial purposes or distribution.

This guidance contains specific recommendations concerning the use of automated modules to completely or partially perform operations for SSRP. It provides more detailed recommendations than previous guidance concerning SSRP. ${ }^{2}$ All operations (synthesis, dilution, sterile filtration and/or vial dispensing) should follow the specific recommendations of the present guidance when performed with an automated module. As much as possible, this guidance offers practical advice, which aims to comply with the requirements of European regulatory bodies. However, this guidance must be interpreted within the legal requirements of each member state. 


\section{Pre-purchase}

Before purchasing a module, a URS should be prepared. This document should contain all the user's requirements related to the module characteristics and performance. Requirements should satisfy the following criteria:

- each requirement should be reviewed, approved and indexed;

- there should be no conflict between requirements;

- each requirement should be testable and subject to verification;

- the URS should be understood and agreed by both user and supplier;

- there should be clear distinction between mandatory/ regulatory requirements and optional features.

Requirements may include, but are not limited to: size, (maximum) weight, yield (expressed in percentage, corrected or non-corrected for decay of radioactivity) for a given radiopharmaceutical, radiochemical purity (e.g.according to a monograph of the Ph.Eur.), compatibility with GMP, cycle time (i.e.time from start of one synthesis to the start of the next, mainly for non-cassette systems with a cleaning procedure), supplies/materials needed (gases, vacuum, cooling, voltage, telepliers, manipulators, dose calibrator, etc.), environmental demands (air class of isolator, air exchange), software characteristics, service/maintenance demands, etc.

\section{Module installation, qualification and maintenance}

\section{Documentation (master document)}

Whatever the origin (commercial or custom made) of the automated module, a master documentation file should be established to gather all records concerning description, installation, initial and periodic qualifications, operating procedures, maintenance and repairs. This file should allow the history of a module to be traced. Deviations during the operating procedures should be classified by criticality (minor, major) and an appropriate action scheme should be proposed in each case.

\section{Hot cell for module installation}

Hot cells for automated module installation should follow the recommendations described in other texts considering the preparation of SSRP. ${ }^{2,4}$ The class of the air within the hot cell must be compatible with operation(s) performed; typically, class C or D for closed operations and class A for open/aseptic operations is required, unless adequate risk assessment has been performed. Potential pitfalls (e.g.cross-contamination, leakage and residual solvents) that can affect the product quality and patient safety should be identified by a risk assessment.

\section{Cleaning before initial installation}

Unless the module has been assembled and shipped in a clean environment, it should be cleaned thoroughly before installation. The module itself must meet the environmental demands for microbiology and particles. This means that the surface should be clean and moving parts (valves, actuators, etc.) should not generate particles.

For multiple-use modules cleaning validation of the chemistry part must be performed. For both types, external surfaces of the mechanical part (and chemistry part for non-disposable device) should be cleaned with suitable detergents and disinfectants, in order to reach a level of chemical and microbiological contaminations compatible with the pharmaceutical class in which the module is to be located. This first cleaning should be carried out in the neighbouring environment of the dedicated area for installation (e.g. near the shielded hot cell). All operations during initial installation should comply with the current procedures used in the area of installation. In particular, general cleaning and transfer SOPs should be followed, in order to introduce the module in the neighbourhood of the dedicated area for installation. During the first cleaning, special attention should be paid to surfaces that will be more difficult to access after installation (e.g. rear and underneath parts). The dedicated area for installation should be cleaned following specific SOPs before the installation of the automated module.

After installation and connection of the module, a second cleaning of accessible external parts of the module and surfaces of the area of installation should be performed following the same SOP as for periodic cleaning between preparation runs.

\section{Initial qualification and periodic qualifications}

Initial qualification and periodic qualification should be planned in the master document describing each automated module.

Initial qualification should include URS, IQ, OQ and PV following general principles and recommendations described in other texts. ${ }^{5} \mathrm{IQ}$ should include verification of the designed module specifications, a check of installed instrumentation and integration of working and maintenance instructions in the master document of the module. The functionalities of the automated module without reagents or chemical components should be checked during $\mathrm{OQ}$. If the module is a commercial unit, the user should ask the supplier to perform a qualification according to internal procedures or to propose a procedure to be performed by the user. If the module is custom made, the user should check that all functionalities meet the specifications included in the master document describing the module. This should include the movement of actuators and the calibration status of the probes (temperature, pressure, and radioactivity). PV of the module should be conducted by performing three complete runs of a representative process covering all normal operations for the concerned preparation process. For example, a module including a preparative chromatographic system should be validated selecting a radiopharmaceutical preparation process, which includes a chromatographic purification. PV should demonstrate that the module is suitable for the intended application in real conditions of use.

Each automated module should follow a programme of periodic qualifications of the probes (temperature, pressure and radioactivity) in order to re-calibrate them if needed. For major updates or repairs of the mechanical part, or in case of major modifications of the control system, a risk assessment should be performed in order to evaluate the potential impact on the process performed with the module. OQ and PV should eventually be performed in accordance with the risk assessment.

\section{Preventative maintenance}

Preventative maintenance should be planned in the master document describing each automated module. Each automated module should follow a programme of preventive maintenance, including the internal mechanical parts of the module (engines, 
seals and moving parts) as well as the chemistry part of any nondisposable system (tubing and valves). The preventive maintenance should include cleaning, lubrication, calibration and, if needed, replacement of components. The schedule of periodic maintenance should be established.

\section{Validation}

\section{Cleaning and cleaning validation}

The cleaning SOP should cover the cleaning of the external surfaces of the mechanical part (for all systems) and the external and internal surfaces of the chemistry part (for non-disposable devices). Disposable chemistry parts do not need to be cleaned if they are intended to be single-use material. However, they must be resistant to materials used in sanitisation prior to transfer into the controlled environment and this must be tested as part of the cleaning validation. Specifications should be established in terms of chemical and microbiological cleanness to be compatible with the environment in which the preparation process takes place.

Validation of the cleaning processes should be performed prior to use of the automated module. The cleaning validation may be process specific, as the cleaning procedures may vary depending on the intended radiochemical process, and the physicochemical nature of the reagents/intermediates/products involved. Validation of external cleaning processes and internal surfaces of the chemistry part (for non-disposable parts of module) should be performed to demonstrate that they are efficient to fulfil the established specifications in the area of effective operation. In particular, the conditioning and storage conditions of a multiple-use chromatographic support (e.g. liquid chromatography column) should demonstrate its efficiency to avoid chemical, radiochemical and microbiological contamination of the final product.

\section{Process validation}

Process validation is process specific and should be conducted as a general process validation including the chemical design and the programmed sequence of operations. A series of three successful validation runs should be performed for the PV of a new sequence of operations (e.g.a new radiosynthesis). To implement a modification of an already in use sequence of operations, a risk assessment should be performed in order to evaluate the potential impact on the process performed with the module. A validation run should be performed with the new sequence in accordance with the risk assessment.

To be successful, a validation run should be performed without deviation from the SOP (e.g. without manual intervention during an automated process). The recorded information should demonstrate that the process occurred normally. The final product obtained during a validation run must comply with all specifications. A validation run must integrate the simultaneous validation of several separate modules (e.g. a synthesis module, a purification module, a dilution module and/or a dispensing module) used in that process. In that case, the performed tests should demonstrate that each module fulfils its specific acceptance criteria (tests for radiochemical and chemical purity will validate a synthesis module, radioactivity concentration measurements will validate a dilution module and radioactivity measurements and sterility tests will validate a dispensing module). If applicable, intermediate sampling should be performed to validate a critical step (e.g. samples of a bulk solution can be taken to perform a bioburden test before sterilization and to validate the pre-filtration step).

\section{Computer and software}

The description of the software used to control an automated module should be inserted in the documentation and updated when necessary. No specific validation is needed for the software itself. However, software characteristics (such as software access management, backup/restore functions, audit trails, data storage, configuration management, etc.) should be subject to validation during $\mathrm{OQ}$, whilst general software suitability, in terms of operational sequence and capability to yield consistent performance, should be subject of validation during PV. Successful chemical and microbiological validation runs performed during qualification of a module should be considered as sufficient to approve the use of a new software release or a specific new sequence.

\section{Media fill test}

Automated dispensing and the accompanying method of sterilization should be initially and periodically validated using microbiological growth medium in place of the bulk radiopharmaceutical solution. The process should demonstrate its efficiency to provide sterile dispensed media. The number of dispensed vials in this test should be the maximum number of vials to be dispensed during a routine run. The vials should be filled with the maximum volume to be dispensed during a routine run.

In the case of single vial filling directly following radiosynthesis and purification steps, a modified sequence mimicking the last transfer operation only should be programmed and used to fill the vial with microbiological growth medium.

\section{Bioburden}

The aim of bioburden testing is to measure the total number of viable microorganisms (total microbial count) of the radiopharmaceutical solution prior to its final sterilization. ${ }^{6}$ Bioburden testing is performed just like a normal run, with or without radioactivity but with the last sterilizing filter removed. In most situations not more than 10 CFUs per $100 \mathrm{~mL}$ will be accepted. ${ }^{7}$

\section{Material requirements}

\section{Mechanical part}

The material used to build the mechanical part should be chosen to be resistant to high radiation doses, to avoid particulate release, to be easily cleaned and to be resistant to detergents and disinfectants used. The description of the materials used should be included in the documentation. Exposed lead without painting, plastic or stainless steel coating should be avoided.

\section{Chemistry part}

All material composing the chemistry system (single-use or nondisposable) should be inert/resistant towards the materials used for synthesis and cleaning, when applicable. In particular, the quality of plastic and metal components should be chosen to avoid release of chemical contaminants into the product. Lubrication of parts in contact with the products of the automated process should be avoided. If necessary, the lubricant must be chosen to fulfil the bio-compatibility criteria and its 
application has to be clearly described. The resistance of materials to radiation and chemicals (reagents and cleaning solutions) should be evaluated in order to establish the schedule of preventive replacement of a non-disposable chemistry part.

\section{Routine runs}

A recording system must be in place in order to assure the traceability of all steps performed during the preparation cycle. Routine use of automated modules should follow a safe cycle of operations. Operations should be organized in order to minimize the risks of cross-contamination between two subsequent batches and to minimize the microbiological contamination of the preparation.

\section{Assembly of dispensing material}

Regardless of whether the dispensing process is a closed or open operation, manual assembly of material (tubing, needles, filters and/or vials) is an open operation and should be conducted in aseptic conditions following published recommendations ${ }^{2,4}$ unless final sterilization of the assembled material follows.

To perform this critical step, operators must wear clothing suitable for the environmental working grade of concern and in any case sterile gloves or gloves cleaned by a detergent/ disinfectant should be worn. The materials used for the assembly of the wetted/concerned part of the dispensing module (and diluting module if dilution is performed in the same area) should be sterile single-use items. Unwrapping should be performed in a manner which minimizes the risk of microbiological contamination of items.

In the case of use of an automatic dispensing system, assembly should be performed preferably in the area in which the module is installed. If assembly takes place in another area, the risk of contamination of the assembly during transfer to this area and during connection to the module should be evaluated and minimized by suitable procedures.

In the case of single vial filling performed directly after radiosynthesis with direct connection to the synthesis module, assembly should be performed in a dedicated area suitable for aseptic open operation. The risk of contamination of the assembly during transfer to the synthesis area and during connection to the synthesis module should be evaluated and minimized by suitable procedures. Transfer and connection should occur after pre-run cleaning and mounting of the synthesis material.

\section{Pre-run cleaning (post-decay cleaning)}

For modules intended to perform radiosynthesis and purification of the radiopharmaceutical (high radioactivity risks), dismantling of the disposable chemistry part or cleaning of the nondisposable chemistry part after use can be delayed for reasons of operator protection. As a consequence, these operations have to be performed following established procedures for radioactive waste disposal, before using the module again. The pre-run cleaning procedure for the non-disposable chemistry part should take place before starting a new preparation of a radiopharmaceutical batch following validated cleaning procedures.

Care should be taken in handling and storage of parts that may have become radioactive by the radiosynthetic processes. Cartridges used in purification steps (e.g. SPE) cartridges for purification of ${ }^{18} \mathrm{~F}$-labelled products), reaction vials and waste container are typical examples of components that may contain long-lived radionuclides. A careful risk assessment should be performed and can be considered part of the cleaning validation procedure.

\section{Mounting of synthesis material}

The operator should wear sterile or sanitized gloves to perform assembly of the synthesis material. For operator convenience as many tests as possible should be performed before introduction of materials and chemicals. In the case of single vial filling performed in the radiosynthesis area, transfer and connection of the assembly for filling should occur after mounting of the synthesis material in order to minimize the risk of microbiological contamination.

\section{Radiosynthesis}

Protection against radiation should be in place before transfer of the radioactivity to the automated module (i.e.the doors of shielded cells should be closed and interlocked with the radionuclide transfer system, if applicable). After radioactivity transfer, the operator initiates the automatic synthesis sequence. The operator should follow the course of the process on the control computer display. Manual intervention should be avoided during the synthesis process. If a mechanical handling system is available in the shielded cell, manual interventions should be reported as deviations in the synthesis report, unless they are normal steps in the process (e.g. manual injection in a preparative HPLC system).

\section{Purification}

Some synthetic processes involve an intermediate purification step by liquid chromatography. The risks of contamination during this step are localized to the injection device, the eluate storage and the fraction collection system. These three parts of the HPLC system should be located in a pharmaceutical environment identical to the one used for the automated synthesis module. Eluents and pumps can be located outside the synthesis cell if all connections between parts are tight. Collection of the eluted fraction of interest is often manually initiated and terminated by the operator based on the information reported by chromatographic detectors.

There are three scenarios in which purification is used. The first involves semi-preparative HPLC purification using pharmaceutical grade eluents (e.g. water, saline or biocompatible buffer \pm ethanol) by which the bulk radiopharmaceutical solution is obtained directly. The second involves semi-preparative HPLC purification with non-pharmaceutical grade eluents (e.g.trifluoroacetic acid and acetonitrile), which must subsequently be removed by evaporation or SPE processing and reformulation. The third involves SPE purification and reformulation. For the critical step of concentration, special attention should be paid to minimize risks of chemical and microbiological contamination. In particular, risk assessment for quality and storage should be established for reagents used after the critical step (see cleaning and cleaning validation).

\section{Formulation}

Formulation is then performed by the module to provide an injectable solution of the radiopharmaceutical. In the case of use of SPE cartridges, a pre-filtration (i.e.using a filter with 
$0.45-\mu \mathrm{m}$ pore size) may be necessary at the end of the synthesis in order to remove particles present before dispensing; however, it must be stressed that this does not constitute sterile filtration. If no further dilution is foreseen, the solution, filtered or not, is the bulk radiopharmaceutical solution.

In case of single vial filling in the radiosynthesis area, the filtered solution is not isolated but directly introduced into the vial using a pre-mounted aseptic assembly connected to the radiosynthesis module. It is not possible to control the quality of this intermediate non-isolated solution during routine synthesis. Validation runs should initially and periodically prove that this solution fulfils specifications that are acceptable to ensure the efficiency of sterilization by filtration in the final vial (bioburden).

\section{Dilution and dispensing}

Sometimes, a dilution process has to take place to adjust the radioactivity concentration at calibration time. The dilution generally proceeds by addition of a dilution solution, mixing and sampling for assay. The dilution process is often performed by a designated module. All these operations should avoid the introduction of particles, chemical and microbiological contaminants into the radiopharmaceutical solution. The result after the dilution step is the bulk radiopharmaceutical solution.

The quality of this intermediate solution should not be controlled during routine preparation. Validation runs should initially and periodically demonstrate that this solution fulfils specifications (especially endotoxin content and bioburden) that are acceptable to ensure the efficiency of the further sterilization (by filtration or using heat). Nevertheless, sampling for the QC tests can be performed at this stage on the final diluted solution.

The dispensing must be performed using a validated method in order to provide accurate activity distribution and to obtain sterile radiopharmaceuticals when final filtration through a $0.22-\mu \mathrm{m}$ filter is performed to yield the final radiopharmaceutical solution. The system should provide a measurement of the radioactivity dispensed into each individual vial. Samples for microbiological control (endotoxins, sterility, retests) and for sample retention may be dispensed as individual vials. The vial (s) for sterility tests should be dispensed at or near the end of the dispensing process. The QC sample must be obtained from the final radiopharmaceutical solution.

Integrity control of the final sterile $0.22-\mu \mathrm{m}$ filter used for dispensing should be performed before the release for use of the finished product. An automated system to decrease filter radioactivity should be considered (rinse with non-radioactive liquid) or an automated filter integrity evaluation system should be integrated in the dispensing module.

\section{Post-run cleaning (pre-decay cleaning)}

For automated synthesis modules, dismantling of the disposable chemistry part or cleaning of the non-disposable chemistry part may not be performed immediately for reasons of operator protection, but should be performed as soon as possible having radiation protection in mind. The cleaning procedure for the mechanical part of the module and for the non-disposable chemistry part should then take place following validated cleaning procedures. If a period of decay of radioactivity is required before cleaning, the delay should be as short as possible, depending on the residual activity on the module and the half-life of the radionuclide (see Pre-run cleaning/post-decay cleaning).

\section{Disclaimers}

This guidance summarizes the views of the Radiopharmacy Committee of the EANM and reflects recommendations for which the EANM cannot be held responsible. The recommendations should be taken in the context of good practice of nuclear medicine and do not substitute for national and international legal or regulatory provisions. The guidance was brought to the attention of the National Societies of Nuclear Medicine.

\section{References}

[1] European Association of Nuclear Medicine Radiopharmacy Committee, Guidelines on current good radiopharmacy practice (cGRPP) in the preparation of radiopharmaceuticals, version 2, 2007. http://www. eanm.org/publications/guidelines/gl_radioph_cgrpp.pdf [23 May 2014].

[2] P. Elsinga, S. Todde, I. Penuelas, G. Meyer, B. Farstad, A. FaivreChauvet, R. Mikolajczak, G. Westera, T. Gmeiner-Stopar, C. Decristoforo, Eur. J. Nucl. Med. Mol. Imaging 2010, 37, 1049. http:// www.eanm.org/publications/guidelines/5_EJNMMI_Guidance_cGRPP fulltext_05_2010.pdf [23 May 2014].

[3] Pharmaceutical Inspection Co-operation Scheme, Document PI 0063: Recommendations on validation master plan, installation and operational qualification, non-sterile process validation, cleaning validation, 2007. http://www.picscheme.org/pdf/25_pi-006-3recommendation-on-validation-master-plan.pdf [23 May 2014].

[4] Pharmaceutical Inspection Co-operation Scheme, Document PE 010-4, Annex 3: Good practices for the preparation of radiopharmaceuticals in healthcare establishments, 2014. http://www.picscheme.org/bo/ commun/upload/document/pe-010-4-guide-to-good-practices-for-thepreparation-of-medicinal-products-in-healthcare-establishments-1.pdf [23 May 2014].

[5] European Commission, Eudralex Volume 4 Good manufacturing practice (GMP) guidelines. Annex 15: Qualification and validation, 2001. http://ec europa.eu/health/files/eudralex/vol-4/pdfs-en/v4an15_en.pdf [23 May 2014].

[6] European Pharmacopoeia, Chapter 2.6.12: microbiological examination of non-sterile products (total viable aerobic count), 2014

[7] European Medicines Agency, Note for guidance on manufacture of the finished dosage form (CPMP/QWP/486/95), 1996, http://www. ema.europa.eu/docs/en_GB/document_library/Scientific_guideline/ 2009/09/WC500002916.pdf [23 May 2014]. 